\title{
matters
}

\section{Natural dynamic backgrounds affect perceived facial dominance}

\begin{abstract}
Alexander Toet
Perception, TNO

We have tested whether natural dynamic backgrounds affect perceived facial dominance. Facial evaluation is based on just two fundamental dimensions of facial appearance: valence and dominance. Perceived facial valence has been shown to be biased towards background valence. However, it is currently unknown, if the perception of facial dominance is also context dependent. In this study, participants rated the perceived dominance of neutral faces superimposed on everyday dynamic backgrounds that were either classified as low (weak) or high (strong) in dominance. Neutral faces were perceived as significantly more dominant when seen on a strong dynamic background than on either a weak or neutral background. Thus, background dominance enhances perceived facial dominance. Since dynamic textures are ubiquitous this finding is relevant for the design and experience of both our daily environment and multimedia content.
\end{abstract}

\section{Objective}

The objective of this study was to investigate whether perceived facial dominance is biased by the dominance of everyday dynamic backgrounds.

\section{Introduction}

People are typically unaware that the context in which they see a face influences their affective judgment, even when this context has absolutely no relevance for their assess-

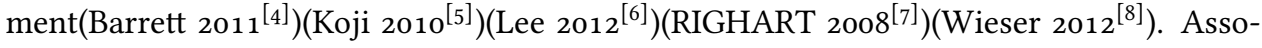
ciations between context and faces are routinely established and modulate face perception already at the early stages of facial feature processing, such that the affective quality of the context transfers to the perceived affective state of a face (Barrett 2011 $\left.{ }^{[4]}\right)\left(\right.$ Wieser 2012 $\left.{ }^{[8]}\right)$ As a result, identical facial configurations may convey strikingly different emotions and dimensional values depending on the context in which they are perceived (Koji 2010 $\left.{ }^{[5]}\right)$ Previous studies shown that perceived facial valence is biased towards background valence; faces perceived in negative (positive) contexts are judged more negative (positive) than in a neutral context $\left(\right.$ Koji 2010 $\left.{ }^{[5]}\right)\left(\right.$ Lee 2012 $\left.{ }^{[6]}\right)$ However, it is currently unknown if the perception of facial dominance is also context dependent. Environmental psychology has shown that the dominance is one of the principle affective qualities of backgrounds and environments. The dominance (sometimes also called strength or potency) of a background is defined as the degree to which it affects the observer(Author B. 2016 $\left.{ }^{[9]}\right)\left(\right.$ Author B. 2016 $\left.{ }^{[10]}\right)$ An observer may feel overwhelmed by and unable to control a strong background, while (s)he may feel in control of- and able to- influence a weak background media. We hypothesized that background dominance may also biased perceived facial dominance. To test this hypothesis, we performed an experiment in which observers judged the dominance of neutral faces presented on natural dynamic textures (spatially repetitive, time-varying visual patterns that repeat or seems to repeat themselves over time (Doretto 2003 ${ }^{[11]}$ ) that were classified as either neutral, low (weak) or high (strong) in dominance (Fig. A). 

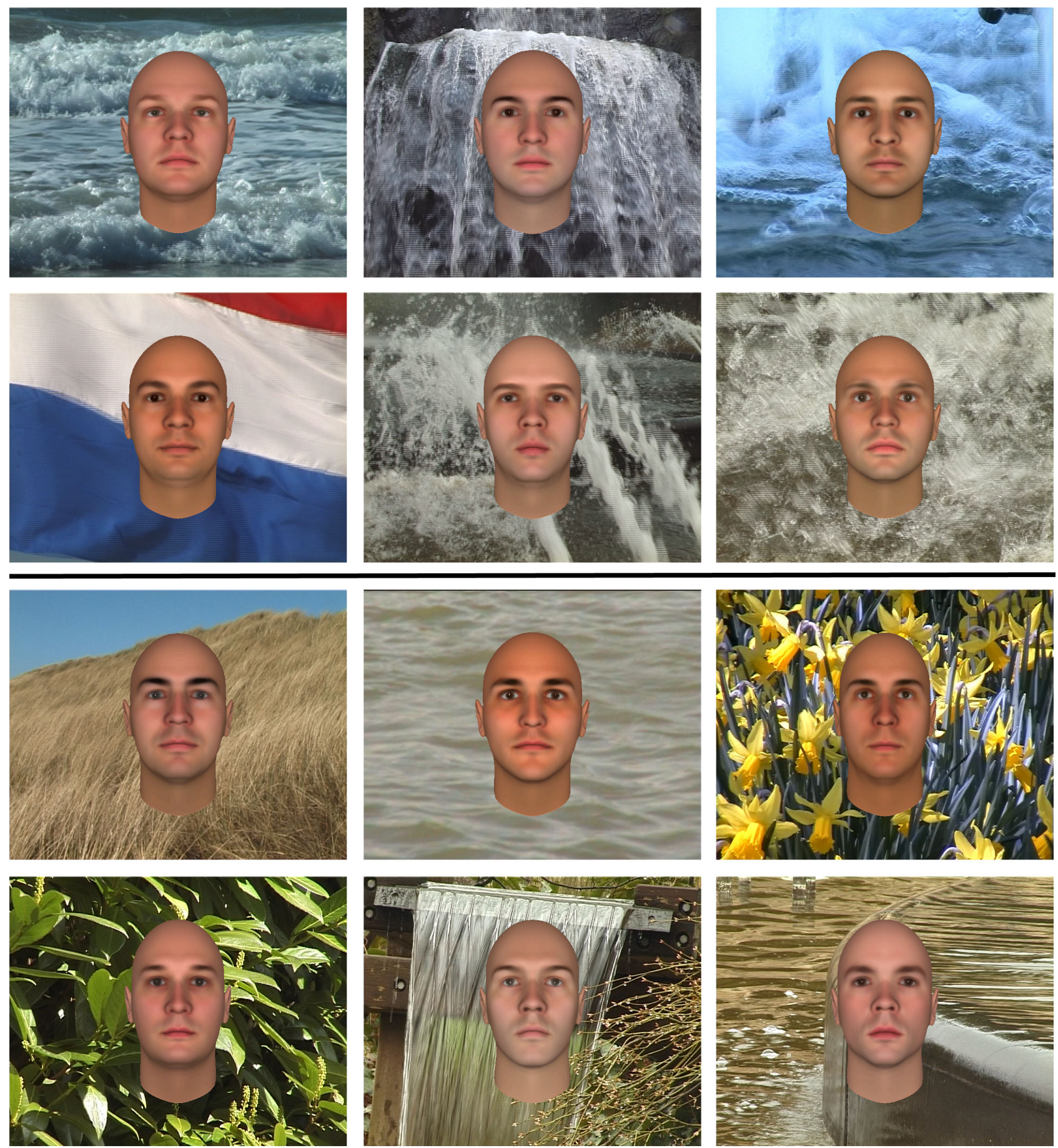

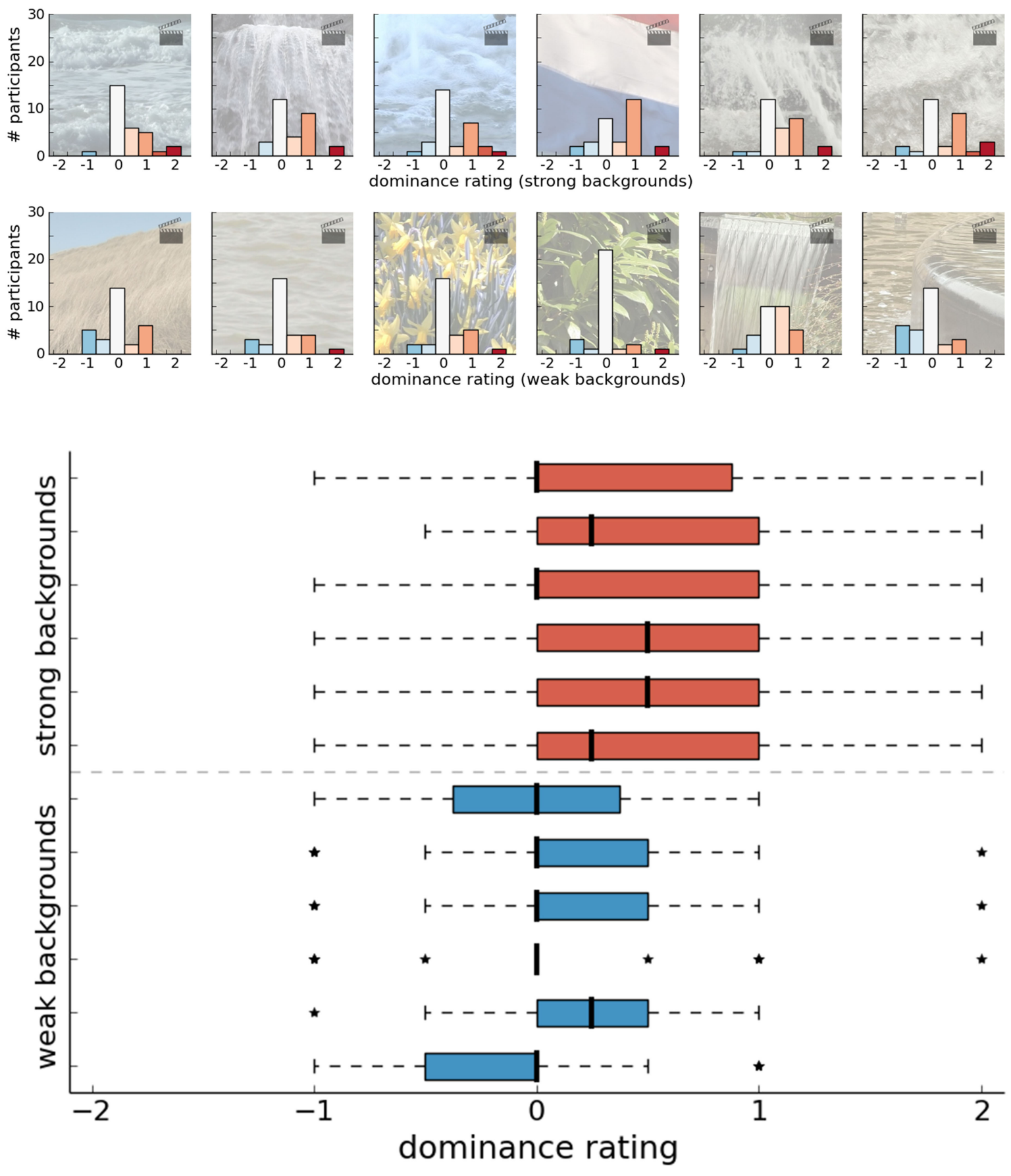

A) The 12 neutral faces from the Princeton faces database(Oosterhof $\left.\left.2008^{[1]}\right)\right]$ overlaid on dynamic textures from the Dyntex database $\left(\right.$ P teri_2010 $\left.{ }^{[2]}\right)$ that are classified as either strong (upper 2 rows) or weak (lower 2 rows) (Toet 2011 $\left.{ }^{[3]}\right)$ B) Median dominance ratings for neutral faces shown on the dynamic textures from the Dyntex databas $q(\mathrm{P}$ teri_$\left.2010^{[2]}\right)$ that are classified as either strong (upper row) or weak (lower row) (Toet 2011 ${ }^{[3]}$ ) In this figure, the background images are shown in lighter shades than those actually used in the experiment.

C) Tukey boxplot of the median dominance ratings for neutral faces shown on respectively the strong (upper six; topdown corresponding to left-right in Fig. B) and weak (lower six) backgrounds. Thick line denotes the median, stars denote outliers (values more than 1.5 IQR below the first quartile or above the third quartile). 


\section{Results \& Discussion}

The results of the experiment were first accumulated by calculating the median dominance rating per background across all 12 faces for each of the 30 participants (Fig. B and C). Next, these median dominance ratings were accumulated across the six strong backgrounds and across the six weak backgrounds individually (again, by using the median). These ratings were then compared with each other and with neutrality (i.e., zero). A Friedman test revealed a significant effect of background on dominance rating $\left.\chi^{2}(2)=13.972, p=0.001\right)$. Next, a post-hoc analysis was performed using multiple Wilcoxon singed rank tests, which were conducted using Bonferroni adjusted alpha levels of 0.0167 per test (0.05/3). The results shows a significant difference between perceived dominance ratings of faces on strong and on weak backgrounds respectively $(Z=3.033, p=0.002)$. Also, perceived dominance ratings for faces on strong backgrounds are significantly different from neutral $(Z=3.305, p=0.001)$. However, perceived dominance ratings for faces on weak backgrounds are not significantly different from neutral $(Z=2.230, p=0.026)$. Neutral faces are perceived as significantly more dominant when seen against a strong background than on either a weak or neutral (dark) background. A Mann-Whitney U-test was performed to test for gender difference. For neutral faces shown on strong backgrounds this test revealed no significant difference between dominance ratings by males $(\mathrm{Md}=0.32, \mathrm{~N}=18)$ and females $(\mathrm{Md}=0.23, \mathrm{~N}=12), \mathrm{U}=86.5, \mathrm{Z}=-0.911, \mathrm{p}=$ $0.368, \mathrm{r}=-0.16$. For neutral faces shown on weak backgrounds there was also no significant difference between males $(\mathrm{Md}=0.08, \mathrm{~N}=18)$ and females $(\mathrm{Md}=0.12, \mathrm{~N}=12), \mathrm{U}=92.0, \mathrm{Z}=-0.678, \mathrm{p}=0.518, \mathrm{r}=-0.12$.

Our current finding that even everyday dynamic textures, such as streaming water, swirling leaves, moving clouds, waving flags or traffic streams can influence perceived facial dominance agrees with the growing body of evidence that background context modulates perceived facial emotions $\left(\right.$ Koji 2010 $\left.{ }^{[5]}\right) \mid\left(\right.$ Lee 2012 $\left.{ }^{[6]}\right)\left(\right.$ RIGHART 2008 $\left.{ }^{[7]}\right)$ The current results also agree with recent brain studies showing that contextual information influences activities in the extended neural network of face processing and thereby alters the perception and evaluation of facial expressions $\left(\operatorname{Schwarz} 2012^{[12]}\right) \mid \operatorname{Van}$ den_Stock_2013 $\left.{ }^{[13]}\right)\left(\right.$ Wieser 2012 $\left.{ }^{[8]}\right)\left(\right.$ Wieser 2014 $\left.{ }^{[14]}\right)$ In particular, it was recently observed that the amygdala integrates facial expression with salient motion information (Hindi Attar_2010 ${ }^{[15]}$ ) This indicates that the amygdala is not only responsive to facial expressions $\left(\right.$ Harris 2012 $\left.{ }^{[16]}\right)$ but also incorporates the overall perceptual context of a stimulus. Hence, it appears that facial evaluation is context dependent and not automatic, hard-wired, effortless and universal as previously proposed(Ekman 1992 $\left.{ }^{[17]}\right)$

In addition to their high prevalence in our daily environment, dynamic textures are also increasingly applied in animation (Lockyer 2012 ${ }^{[18]}$ ) and video synthesis(Doretto 2003 ${ }^{[11]}$ ) and are deployed on large-scale digital billboards and electronic wallpaper(Huang $2005^{[19]}$ ) Because of this ubiquity of dynamic textures, our current findings may be relevant for the design and experience of both our daily environment and multimedia content.

\section{Conclusions}

Everyday dynamic background textures such as streaming water, swirling leaves, moving clouds, waving flags or traffic streams can bias perceived facial dominance.

\section{Limitations}

A limitation of this study is the small number of stimuli (faces and backgrounds) that were tested. Future studies should use a larger number of dynamic background textures with widely varying content and motion patterns, covering the entire dominance range, to allow a closer investigation (correlation) of the relation between background dominance and perceived facial dominance. In addition, artificial affective motion textures with well specified path curvature, speed and texture layou (Lockyer 2012 $\left.{ }^{[18]}\right)$ may serve to systematically investigate the relation between different spatio-temporal texture parameters and perceived facial dominance.

\section{Conjectures}

We expect that visual background dominance may also bias the affective appraisal of non-face objects with no evident semantic affective connotation. Moreover, it is also likely that this effect may carry over to other sensory modalities (Schreuder 2016 $\left.{ }^{[20]}\right)$ Hence, a dominant visual background may bias the perception of certain smells, tastes or sounds. We plan to investigate these issues in a follow-up study. 


\section{Additional Information}

Methods and Supplementary Material

Please see https://sciencematters.io/articles/201610000018.

\section{Ethics Statement}

The participants read and signed an informed consent prior to the experiment. The experimental protocol was reviewed and approved by the TNO Ethics Committee and was in accordance with the Helsinki Declaration of 1975, as revised in $2013\left(2013^{[21]}\right)$

\section{Citations}

[1] N. N. Oosterhof and A. Todorov. "The functional basis of face evaluation". In: Proceedings of the National Academy of Sciences 105.32 (Aug. 2008), pp. 11087-11092. DoI: 10 . 1073/pnas . 0805664105 URL: https : / / doi . org / 10 . $1073 \%$ 2Fpnas.0805664105.

[2] Renaud Péteri, Sándor Fazekas, and Mark J. Huiskes. "DynTex: A comprehensive database of dynamic textures". In: Pattern Recognition Letters 31.12 (Sept. 2010), pp. 1627-1632. DOI: 10 . 1016/ j.patrec.2010.05.009 urL: https://doi.org/ $10.1016 \% 2 \mathrm{Fj}$.patrec.2010.05.009

[3] Alexander Toet et al. "Emotional effects of dynamic textures". In i-Perception 2.9 (2011), pp. 969-991. DOI: 10 . 1068/i0477 URL: https://doi.org/10.1068\%2Fi0477

[4] Lisa Feldman Barrett, Batja Mesquita, and Maria Gendron. "Context in Emotion Perception". In: Current Directions in Psychological Science 20.5 (Oct. 2011), pp. 286-290. DOI: 10 . 1177/ 0963721411422522 uRL: https : / / doi . org / 10 . 1177\%2F0963721411422522

[5] Shahnaz Koji and Myra Fernandes. "Does it matter where we meet? The role of emotional context in evaluative first impressions." In: Canadian fournal of Experimental Psychology/Revue canadienne de psychologie expérimentale 64.2 (2010), pp. 107-116. DOI: 10 . 1037 / a0019139 uRL: https : / / doi . org / 10.1037\%2Fa0019139

[6] Tae-Ho Lee, June-Seek Choi, and Yang Seok Cho. "Context Modulation of Facial Emotion Perception Differed by Individual Difference". In: PLoS ONE 7.3 (Mar. 2012). Ed. by Alessio Avenanti, e32987. Dor: 10 . 1371/ journal . pone . 0032987 URL https : //doi.org/10.1371\%2Fjournal . pone. 0032987 .

[7] R. RIGHART and B. DE GELDER. "Recognition of facial expressions is influenced by emotional scene gist". In: Cognitive, Affective, \& Behavioral Neuroscience 8.3 (Sept. 2008), pp. 264-272. DOI: 10.3758/cabn .8.3.264 urL: https://doi.org/ $10.3758 \% 2 \mathrm{Fcabn} .8 .3 .264$

[8] Matthias J. Wieser and Tobias Brosch. "Faces in Context: A Review and Systematization of Contextual Influences on Affective Face Processing". In: Frontiers in Psychology 3 (2012). DoI: 10 . 3389/fpsyg . 2012.00471. uRL:https : //doi .org/ 10.3389\%2Ffpsyg.2012.00471

[9] Mehrabian and A. "Basic dimensions for a general psychological theory". In: Cambridge and MA: Oelgesschlager and Gunn and and Hain (1980).

[10] Mehrabian et al. "A verbal measure of information rate for studies in environmental psychology." In: Environment and Behavior 6 (1974), pp. 233-252.

[11] Doretto et al. "Dynamic textures". In: International fournal of Computer Vision 51(2) (2003), pp. 91-109.
[12] Katharina A. Schwarz et al. "Why are you looking like that? How the context influences evaluation and processing of human faces". In: Social Cognitive and Affective Neuroscience 8.4 (Jan. 2012), pp. 438-445. DOI: 10 . 1093/scan/nss013 URL: https : //doi.org/10.1093\%2Fscan\%2Fnss013

[13] Jan Van den Stock et al. "How affective information from faces and scenes interacts in the brain". In: Social Cognitive and Affective Neuroscience 9.10 (Aug. 2013), pp. 1481-1488. Dor:10 . 1093/ scan / nst 138 uRL: https : / / doi . org / 10 . 1093\% 2Fscan\%2Fnst 138 .

[14] Matthias J. Wieser et al. "Not so harmless anymore: How context impacts the perception and electrocortical processing of neutral faces". In: NeuroImage 92 (May 2014), pp. 74-82. DoI: 10 .1016/ j . neuroimage . 2014.01.022 uRL: https : / / doi. org/10.1016\%2Fj.neuroimage.2014.01.022

[15] C. Hindi Attar et al. "Emotional Processing in a Salient Motion Context: Integration of Motion and Emotion in Both $\mathrm{V}_{5} / \mathrm{hMT}+$ and the Amygdala”. In: Fournal of Neuroscience 30.15 (Apr. 2010), pp. 5204-5210. Dor: 10 . 1523 / jneurosci. 5029 09 . 2010 urL: https : / / doi . org / 10 . $1523 \%$ 2Fjneurosci.5029-09.2010

[16] R. J. Harris, A. W. Young, and T. J. Andrews. "Morphing between expressions dissociates continuous from categorical representations of facial expression in the human brain". In: Proceedings of the National Academy of Sciences 109.51 (Dec. 2012), pp. 2116421169. DoI: 10 . 1073 / pnas . 1212207110 URL: https : //doi.org/10.1073\%2Fpnas.1212207110

[17] Paul Ekman. "An argument for basic emotions”. In: Cognition \& Emotion 6.3 (May 1992), pp. 169-200. DOI: 10 . 1080/ 02699939208411068 urL: https : / / doi . org/10 . 1080\%2F02699939208411068

[18] Matt Lockyer and Lyn Bartram. "Affective motion textures". In: Computers \& Graphics 36.6 (Oct. 2012), pp. 776-790. DOI: 10 . $1016 / \mathrm{j}$.cag . 2012.04 .009 uRL: https : / / doi . org/10.1016\%2Fj.cag.2012.04.009

[19] Jeffrey Huang and Muriel Waldvogel. "Interactive wallpaper". In: Proceedings of the ACM SIGGRAPH o5 electronic art and animation catalog on - GRAPH 'o5. Association for Computing Machinery (ACM), 2005. DOI: 10 . 1145/1086057 . 1086142 URL: https://doi.org/10.1145\%2F1086057.1086142

[20] Eliane Schreuder et al. "Emotional Responses to Multisensory Environmental Stimuli”. In: SAGE Open 6.1 (Jan. 2016), p. 215824401663059 . DOI: 10 . $1177 / 2158244016630591$. uRL: https : / / doi . org / 10 . $1177 \%$ 2F2158244016630591.

[21] "World Medical Association Declaration of Helsinki". In: JAMA 310.20 (Nov. 2013), p. 2191. DoI: 10 . $1001 /$ jama . 2013 . 281053 URL: https : / /doi.org/10.1001\%2Fjama . 2013.281053 
[22] Alexander Toet and Susanne Tak. "Look out, there is a triangle behind you! The effect of primitive geometric shapes on perceived facial dominance". In: i-Perception 4.1 (2013), pp. 53-56. DoI: 10 . 1068/i0568sas urL: https ://doi .org/10 .1068\% 2Fi0568sas

[23] Rebecca E. Cooney et al. "Amygdala activation in the processing of neutral faces in social anxiety disorder: Is neutral really neutral?” In: Psychiatry Research: Neuroimaging $14^{8.1}$ (Nov. 2006), pp. 55-
59. DOI: $10.1016 / \mathrm{j}$.pscychresns. 2006.05 .003 URL: https : //doi.org/10.1016\%2Fj.pscychresns . 2006.05 .003

[24] K. Lira Yoon and Richard E. Zinbarg. "Interpreting neutral faces as threatening is a default mode for socially anxious individuals." In: Journal of Abnormal Psychology 117.3 (2008), pp. 680-685. DOI: $10.1037 / 0021-843 x \cdot 117.3 .680$ uRL: https : / / doi.org/10.1037\%2F0021-843x.117.3.680 